Aim of the study: This study aims to characterise alterations in the immunohistochemical expression of p27 in normal tissue of the salivary gland surrounding pleomorphic adenoma and in the tumour cells of pleomorphic adenoma.

Material and methods: A retrospective study of data including 120 cases of pleomorphic adenomas ( 66 female, 54 male) retrieved from the files of the Oral Pathology Departments of Aleppo University, Syria and Al-Farabi Dental and Nursing College. Immunohistochemical expression against p27 was examined in the selected cases. The percentage of p27-positive nuclei was semi-quantitatively assessed by two independent observers and scores were given. The statistical analysis included the use of descriptive statistics and proportional frequencies.

Results: The results showed that P27 nuclear staining with high staining (moderate to strong positive staining in more than $50 \%$ of nuclei, depending on the percentage of nuclei for the cells) was noted in tumour duct cells of pleomorphic adenoma in 90 (75\%) cases out of 120 , while $30(25 \%)$ cases showed low staining (positive staining in less than $5 \%$ of nuclei, depending on the percentage of nuclei for the cells).

Conclusions: Our data suggests that p27 might have a minor role in the development of pleomorphic adenoma.

Key words: pleomorphic adenoma, p27 expression, immunostaining, tumour suppressor gene, nuclear staining.

Contemp Oncol (Pozn) 2015; 19 (1): 28-31 DOI: $10.5114 /$ wo.2014.45294

\section{P27 expression in pleomorphic salivary gland adenoma}

\author{
Bassel Tarakji1, Ayesha Umair ${ }^{1}$, Mohammed Alsakran Altamimi², \\ Saleh Nasser Azzeghaiby', Abla Sayed Mahmoud ${ }^{1}$, Shourouk Darwish ${ }^{1}$, \\ Mohammad Zakaria Nassani ${ }^{2}$, Nipun Ashok ${ }^{1}$
}

${ }^{1}$ Department of Oral Maxillofacial Sciences, Al-Farabi College of Dentistry and Nursing, Riyadh, Saudi Arabia

2Department of Restorative Dentistry, Al-Farabi College of Dentistry and Nursing Riyadh, Saudi Arabia

\section{Introduction}

The discovery of useful molecular markers involved in crucial steps of development of salivary gland tumours has played a major role in tumour diagnosis and staging. Human cancer, manifested as uncontrolled cellular proliferation, is commonly caused by changes in the expression or activity of proteins involved in regulating the normal cell cycle [1]. p27, a member of the cip/kip family of cyclin-dependent kinase inhibitors, is expressed at high levels in GO cells and promotes cell arrest and apoptosis. The protein that specifically binds and targets p27 for degradation is S-phase kinase-associated protein-2 (skp-2), which may be the main rate-limiting regulator for p27 degradation [1]. Increase in p27 is associated with cell growth arrest, cell differentiation, or an increase in apoptotic activities whereas decreased p27 expression is related to increased proliferation and tumorigenesis [2, 3].

Pleomorphic adenoma (PA) is the most common neoplasm of the salivary glands, and in some cases it has shown a tendency to undergo malignant transformation in its natural course [4]; therefore, it is necessary to evaluate the molecular events in PA. The role of p27 in salivary glands, particularly in PAs, is unclear. The purpose of this study is to determine the alteration in the immunohistochemical expression of p27 in the tumour cells of PAs. Also, we have to assess the correlation of age, gender, size of tumour, and p27 expression.

\section{Material and methods}

Retrospective study data including 120 cases of PAs (66 female, 54 male) were retrieved from the files of the Oral Pathology Departments of Aleppo University, Syria and Al-Farabi Dental and Nursing College, Riyadh. Normal tissue of the salivary gland surrounding the tumour was used as a control. Additionally, p27 immunohistochemical expression against p27 was examined in the selected cases. Microscopic slides stained with haematoxylin and eosin were reviewed by two pathologists to confirm the histopathological diagnosis and to reclassify the studied cases. P27 expression in normal tissue of the salivary gland and PA will be evaluated to make a comparison between these two fields. Nuclear staining of the duct and acinar cells will be assessed in normal tissue of the salivary gland to make a comparison between p27 expression in normal tissue components. P27 expression in tumour duct cells and myxochondroid tissue of our cases will be evaluated to find out whether or not the duct cells are responsible for the development of pleomorphic adenoma depending on the disease progression. Most of the pleomorphic adenoma cases were from the parotid gland (100 out of 120 cases) and minor salivary gland (20 out of 120 cases). 
Ethical approval was given by the Ethics Committee of the Research Department of Al-Farabi College of Dentistry and Nursing, Riyadh, Saudia. Reference to be used: (AEC:3-014).

Immunostaining for p27 Kip1 protein was achieved by using a commercially available antibody (Transduction Laboratories, Lexington, KY) and streptavidin-biotin kit (Nichirei, Japan). Deparaffinised tissue sections were immersed in methanol containing $0.3 \%$ hydrogen peroxide for 30 minutes to block endogenous peroxidase activity. Microwave in citrate buffer was performed for 3 times for 10 minutes to retrieve the antigenicity. The sections were then incubated with normal rabbit serum for 30 minutes to block nonspecific antibody binding sites. The sections were treated consecutively at $4^{\circ} \mathrm{C}$ with anti-p27Kip1 monoclonal antibody (diluted 1 : 100) overnight. This was followed by treatment with biotinylated anti-mouse immunoglobulin and $\mathrm{G}$ rabbit serum for 30 minutes, and then by streptavidin-biotinylated horseradish peroxidase complex for another 30 minutes. Peroxidase staining was performed for 5-10 minutes using a solution of diaminobenzidine tetrahydrochloride in $50 \mathrm{~mm}$ Tris- $\mathrm{HCl}(\mathrm{pH} 7.5)$ containing $0.001 \%$ hydrogen peroxide. The sections were weakly counterstained with Mayer haematoxylin.

A brown precipitate seen within the nucleus confirmed the presence of p27 protein. A total of five areas were chosen randomly from each of the tested slides and scored at high power magnification. The nuclear staining was observed exclusively in the nuclei of the test cells. The positive control was normal salivary gland tissue adjacent to the tumour specimens. The negative control was included for each staining using normal goat serum instead of primary antibodies. None of the negative controls displayed brown staining in the test cells. The percentage of p27 positive nuclei was semi-quantitatively assessed by two independent observers and scores were given. The immunostained sections were scored as negative staining, low staining, and high staining depending on the percentage of nuclei that showed positive staining according to the following scheme [3]:

- negative staining = positive staining in less than $5 \%$ of nuclei,

- low staining $=$ moderate to strong positive staining in $5 \%$ to $50 \%$ of nuclei,

- high staining = moderate to strong positive staining in more than $50 \%$ of nuclei.

The statistical analysis included the use of descriptive statistics and frequency proportions. All statistical tests were two-sided, and $p$-values less than 0.05 were considered to be statistically significant.

\section{Results}

P27 expression of the nuclear staining was studied in areas of the PA of salivary glands. The results (nuclear staining of duct cells) indicated that p27 showed high positive nuclear staining in 118 (98.3\%) cases out of 120 and low staining in 2 cases (1.6\%).

P27 nuclear staining of the acinar cells showed negative staining in $25(20.8 \%)$ cases out of 120 , and there were 35 (29.1\%) with low staining and 60 (50\%) with high staining. The existence of p27 positive expression was higher in non-duct tumour cells than acinar cells.

P27 nuclear staining with high staining was noted in tumour duct cells of PA in 90 (75\%) cases out of 120 (Fig. 1), while 30 (25\%) cases showed low staining (Fig. 2, Table 1). P27 nuclear staining in myxochondroid tissue was identified in $98(81.6 \%)$ cases with high staining, while $22(18.3 \%)$ cases had low staining.

\section{Discussion}

Our results showed that p27 was expressed in both the normal duct cells and the acinar cells. These results are similar to those obtained by Takata et al. [5], who mentioned that p27Kip1 protein was consistently detected in the nuclei of ductal and acinar cells of non-neoplastic salivary glands adjacent to the salivary adenoid cystic carcinoma.

Zhu et al. [6] indicated that the current histogenic theory of salivary gland tumourigenesis describes the acinar cells as functionally mature cells, and they suggested that the acinar cells were terminally differentiated and incapable of further proliferation. This theory considered that proliferation for the purpose of repair and regeneration was confined to stem cells residing exclusively amongst luminally located intercalated duct cells or basally located excretory duct cells. The results of our work are consistent with this theory as p27 expression shows significant difference in the non-tumour duct compared with acinar cells in the normal tissue surrounding the PA.

P27 expression in PA showed that the incidence of aberrant expression of these proteins was higher in tumour duct cells than in myxochondroid tissue. Our results are in agreement with others. Takata et al. [4], found that p27 was expressed in all cases of PA. Akrish et al. [7], indicated that all cases of PA $(n=6)$ strongly expressed p27. Choi et al. [8] indicated that there are a variety of p27kip1 expressions depending on the disease progression, aggressiveness, recurrence, and metastasis. Furthermore, it was demonstrated that there was a correlation between low level of p27Kip1 expression and high-grade mucoepidermopid carcinoma, large tumour size, positive nodal status, advanced clinical stage, and better prognosis. Some researchers tried to discover the genetic alterations of p27Kip1 in human tumours, but unlike other CDKIs, p16, and p21 the genetic mutation of p27kip1 is extremely rare [9]. Instead, regulation of the p27kip1 protein appears to

Table 1. Expression of duct cells surrounding pleomorphic adenoma and pleomorphic adenomas

$\begin{array}{lcc}\text { P27 expression in non-tumour duct cells } & \text { high positive nuclear staining } & \text { low staining } \\ & 118(98.3 \%) & 2(1.6 \%) \\ \text { P27 expression in tumour duct cells } & \text { high positive nuclear staining } & \text { low staining } \\ & 90(75 \%) & 30(25 \%)\end{array}$




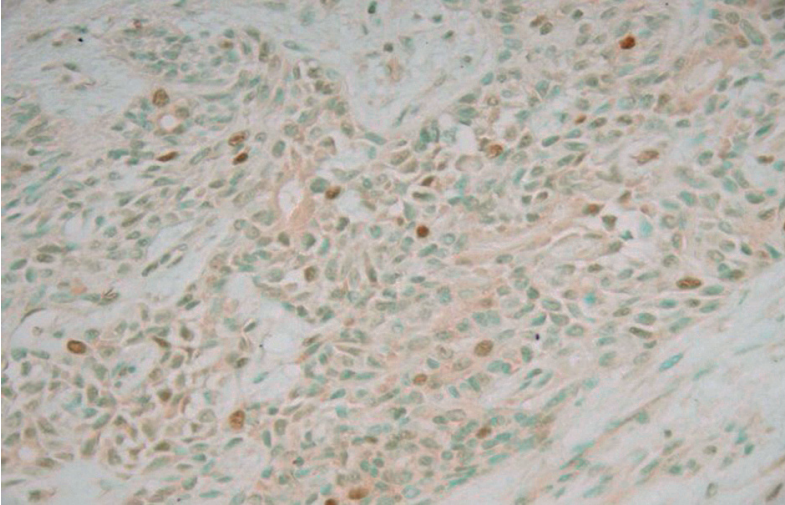

Fig. 1. P27 nuclear staining with high staining in tumour duct cells of pleomorphic adenoma

occur primarily at the posttranslational level by ubiquitin-mediated proteasomal proteolysis pathway [10, 11]. Although it was difficult to follow up the patients who had undergone surgery, our results indicated that there was no correlation between age, gender, size of tumour, and expression of p27 in PAs. The evolution time of the tumour is one of the important factors to be evaluated; unfortunately it was not considered in this study because of the difficulties in following up our large sample (120 cases). Aoyagi et al. [12] mentioned that p27 was correlated with lymph node metastasis, depth of invasion, and proliferative activity of gastric cancer, hence p27 was a useful predictive marker of prognosis of gastric cancer.

Gezginc et al. [13] reported that p27 immunoreactivity decreases progressively as lesions progress from proliferative benign endometrium to frank carcinoma.

Gao et al. [14] mentioned that low p27 expression has a poor prognosis in patients with oral squamous cell carcinoma. In this study we tried to modify the criteria of assessment of staining to avoid any confusion in the interpretation of the results. In our previous publication [15-17] we used different criteria such as the following: negative staining, low staining, moderate staining, and strong staining. We think that the use of different criteria in literature for the evaluation of intensity of staining causes differences in the interpretation of the results, so it would be logical to use only one criterion instead. The used criteria of negative staining, low staining, and high staining score will give a better evaluation of immunostaining because the used criteria make no significant difference in moderate and strong staining. Although the reliability of immunostaining is good, it is recommended that it is combined with polymerase chain reaction technique to identify all the cells that show abnormal expression. Although pleomorphic adenomas are benign, they can show a malignant form in minor salivary glands [18]. Our results were that 30 out of 120 cases (25\%) showed low staining in the duct cells of PA as compared with 2 cases (1.6\%) in non-tumour duct cells. This study shows that p27 expression is reduced in some cases of pleomorphic adenoma.

In conclusion, low expression of P27 was detected within PA in $25 \%$ of cases in our study; we can conclude that p27 may have some role in the development of PA.

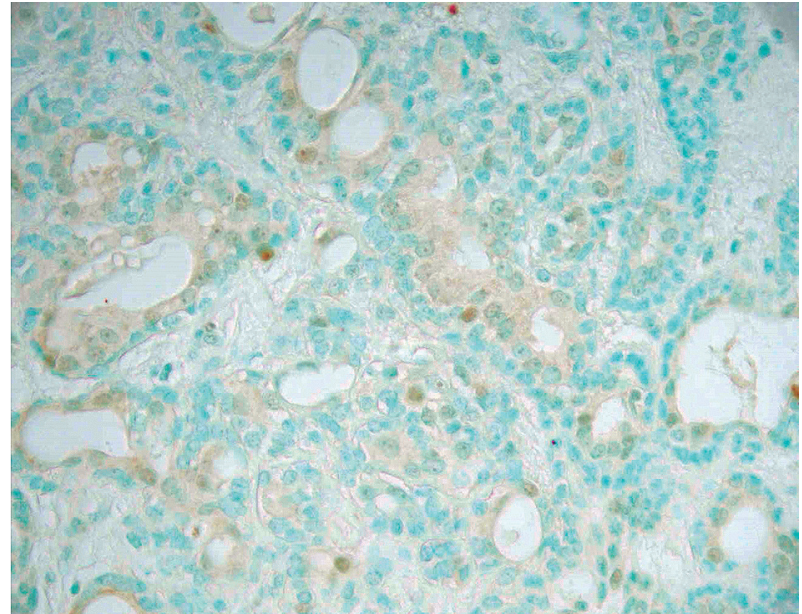

Fig. 2. P27 nuclear staining with low staining in tumour duct cells of pleomorphic adenoma

The authors declare no conflict of interest.

\section{References}

1. Shapira M, Ben-Izhak O, Linn S, Futerman B, Minkov I, Hershko DD. The prognostic impact of the ubiquitin ligase subunits Skp2 and Cks1 in colorectal carcinoma. Cancer 2005; 103: 1336-46.

2. Said TK, Moraes RC, Singh U, Kittrell FS, Medina D. Cyclin-dependent kinase (cdk) inhibitors/cdk4/cdk2 complexes in early stages of mouse mammary preneoplasia. Cell Growth Differ 2001; 12: 285-95.

3. Pillay K, McCleod H, Chetty R, Hall P. A study to investigate the role of p27 and cyclin E immunoexpression as a prognostic factor in early breast carcinoma. World J Surg Oncol 2011; 9: 31.

4. Di Palma S. Carcinoma ex pleomorphic adenoma, with particular emphasis on early lesions. Head Neck Pathol 2013; 7 Suppl 1: S6876 .

5. Takata T, Kudo Y, Zhao M, Ogawa I, Miyauchi M, Sato S, Cheng J, Nikai $H$. Reduced expression of p27(Kip1) protein in relation to salivary adenoid cystic carcinoma metastasis. Cancer 1999; 86: 928-35.

6. Zhu Q, White FH, Tipoe GL. The assessment of proliferating cell nuclear antigen (PCNA) immunostaining in human benign and malignant epithelial lesions of the parotid gland. Oral Oncol 1997; 33: 29-35.

7. Akrish S, Ben-Izhak O, Peled M. P27/SKP-2 histochemical profile is relevant to malignant salivary gland tumors (MST) histogenesis and tumorgrade. Head Neck Pathol 2012; 6: 157-65.

8. Choi CS, Choi G, Jung KY, Choi JO, Chae YS. Low expression of p27(Kip1) in advanced mucoepidermoid carcinomas of head and neck. Head Neck 2001; 23: 292-7.

9. Kawamata N, Morosetti R, Miller CW, et al. Molecular analysis of the cyclin-dependent kinase inhibitor gene p27/Kip1 in human malignancies. Cancer Res 1995; 55: 2266-9.

10. Loda M, Cukor B, Tam SW, Lavin P, Fiorentino M, Draetta GF, Jessup JM, Pagano M. Increased proteasome-dependent degradation of the cyclin-dependent kinase inhibitor p27 in aggressive colorectal carcinomas. Nat Med 1997; 3: 231-4.

11. Pagano M, Tam SW, Theodoras AM. Role of the ubiquitin-proteasome pathway in regulating abundance of the cyclin-dependent kinase inhibitor p27. Science 1995; 269: 682-5.

12. Aoyagi K, Kouhuji K, Miyagi M, Imaizumi T, Kizaki J, Isobe T, Shirouzu K. Expression of p27Kip1 protein in gastric carcinoma. Hepatogastroenterology 2013; 60: 390-4.

13. Gezginc ST, Celik C, Dogan NU, Toy H, Tazegul A, Colakoglu MC. Expression of cyclin A, cyclin E and p27 in normal, hyperplastic and 
frankly malignant endometrial samples. J Obstet Gynaecol 2013; 33: 508-11.

14. Gao L, Gu W, Zheng J, et al. Clinicopathalogical and prognostic significance of p27 expression in oral squamous cell carcinoma: a meta-analysis. Int J Biol Markers 2013; 28: e329-35.

15. Tarakji B, Baroudi K, Darwish S, Sakka S, Hanouneh S. Immunohistochemical expression of p16 in pleomorphic salivary adenoma. Turk Patoloji Derg 2013; 29: 36-40.

16. Tarakji B, Alenzi F, Al-Khuraif AA. Assessment of inverse correlation of $\mathrm{p} 16$ and $\mathrm{pRb}$ expression in carcinoma ex pleomorphic adenoma. Pol J Pathol 2013; 64: 144-8.

17. Tarakji B, Baroudi K, Hanouneh S, Nassani MZ, Alotaibi AM, Kharma MY, Azzeghaiby SN. Expression of p21 is dependent on or independent of p53 in carcinoma ex pleomorphic adenoma (undifferentiated and adenocarcinoma types). Pol J Pathol 2012; 63: 286-91.

18. Rahnama M, Orzędała-Koszel U, Czupkałło L, Lobacz M. Pleomorphic adenoma of the palate: a case report and review of the literature. Contemp Oncol (Pozn) 2013; 17: 103-6.

\section{Address for correspondence}

\section{Bassel Tarakji}

Alfarabi Dental School

e-mail: denpol@yahoo.co.uk

Submitted: 2.07 .2014

Accepted: $\quad 7.08 .2014$ 